\title{
ANÁLISE DA EFETIVIDADE DE ESTRATÉGIA NUTRICIONAL EM MULHERES COM SÍNDROME METABÓLICA
}

\section{Claudia Brauna}

Nutricionista graduada no curso de Nutrição da Universidade Estadual do Centro Oeste, Guarapuava/PR Brasil.

Rafaely de Fatima Fernandes Almeida Vieira

Acadêmica do curso de Nutrição da Universidade Estadual do Centro Oeste, Guarapuava/PR, Brasil.

\section{Adriana Masiero Kuhl}

Mestre em Ciências da Saúde pela UFSC/SC, Docente do curso de Nutrição, Universidade Estadual do Centro Oeste, Guarapuava/PR, Brasil.

Angélica Rocha de Freitas Melhem

Doutora em Ciências pela UNIFESP/SP, Docente do curso de Nutrição, Universidade Estadual do Centro Oeste, Guarapuava/PR, Brasil.

\section{Dalton Luiz Schiessel}

Doutor em Ciências Biológicas e da Saúde pela UFPR/ PR, Docente do curso de Nutrição, Universidade Estadual do Centro Oeste, Guarapuava/PR, Brasil

\section{Gabriela Datsch Bennemann}

Doutora em Ciências Agrárias pela UNICENTRO/PR, Docente do curso de Nutrição, Universidade Estadual do Centro Oeste, Guarapuava/PR, Brasil.

\section{Mariana Abe Vicente Cavagnari}

Doutora em Ciências da Saúde pela UNIFESP/SP, Docente do curso de Nutrição, Universidade Estadual do Centro Oeste, Guarapuava/PR, Brasil.
RESUMO: O objetivo deste estudo foi analisar a efetividade de uma estratégia nutricional em mulheres obesas com Síndrome Metabólica (SM). Estudo longitudinal caso-controle estruturado com dois grupos, sendo mulheres obesas com diagnóstico clínico de SM (Grupo Caso) e mulheres obesas sem diagnóstico de SM (Grupo Controle), em acompanhamento nutricional na Clínica Escola de Nutrição da Unicentro. A coleta de dados ocorreu em duas etapas, com intervalos de um mês, sendo realizadas medidas antropométricas, análise do consumo alimentar e exames bioquímicos. A estratégia nutricional utilizada foram orientações nutricionais e plano alimentar individualizado. Foram avaliadas 20 mulheres, cada grupo com número equivalente. Pacientes com SM apresentaram valores de CC e glicemia em jejum superiores ao grupo controle $(p<0,005)$, ingestão de ácidos graxos acima do recomendado. A intervenção nutricional foi efetiva em pacientes com SM, demonstrando em curto prazo melhora da qualidade da alimentação e do perfil sérico glicêmico e lipídico, redução de medidas antropométricas.

PALAVRAS-CHAVE: Antropometria; Dislipidemias; Nutrição; Obesidade.

\section{ANALYSIS OF THE EFFICACIOUSNESS OF NUTRITIONAL STRATEGY IN FEMALES WITH METABOLIC SYNDROME}

\begin{abstract}
The efficaciousness of nutritional strategy in obese females with metabolic syndrome (MS) is evaluated. Current case-controlled longitudinal study was structured on two groups, with obese women with a clinical diagnosis of MS (Case Group) and obese females without MS (Control Group), within the context of nutritional follow-up in the Clinical Nutritional School of the Unicentro. Data were collected in two stages, at a month's interval, with anthropometric measurements, analysis of food consumption and biochemical tests. Nutritional strategy comprised nutritional supervision and personal food planning. Twenty females were assessed for each group. MS patients had CC and glycaemia rates higher than those of control group $(p<0.005)$ and ingestion of fatty acids above that recommended. Nutritional intervention was effective in patients with MS and revealed that in a short time there was an improvement in food quality, in glycemic and lipid serum and in anthropometric measurements.
\end{abstract}

KEY WORDS: Anthropometry; dyslipidemia; nutrition; obesity. 


\section{INTRODUÇÃO}

A obesidade é uma das crescentes epidemias do século XXI com cerca de 2 bilhões de adultos com excesso de peso ou obeso ${ }^{1}$. Essa condição está atrelada à crescente urbanização que influenciou nos hábitos de vida sedentários, aumento no consumo de alimentos industrializados e calóricos ${ }^{2}$.

Paralelo ao aumento da obesidade, tem-se a Síndrome Metabólica (SM), sendo estimada prevalência global de até $25 \%$ na população, representando $7 \%$ da mortalidade geral e $17 \%$ das mortes associadas com doenças cardiovasculares ${ }^{3}$. A SM é definida como um conjunto de fatores de risco cardiometabólicos, que compreendem obesidade abdominal, dislipidemia, hiperglicemia e aumento dos níveis pressóricos, sendo considerada importante preditora de doença cardiovascular, diabetes mellitus e todas as causas de mortalidades futuras ${ }^{2,4,5}$.

De acordo com a Organização Mundial de Saúde (OMS), os fatores de risco mais importantes para a morbimortalidade relacionada às Doenças Crônicas Não Transmissíveis (DCNT) são a hipertensão arterial sistêmica, a hipercolesterolemia, a ingestão insuficiente de frutas, as hortaliças e leguminosas, o sobrepeso ou obesidade, a inatividade física e tabagismo ${ }^{6}$. Cinco desses fatores de risco estão relacionados à alimentação e à atividade física e três deles têm grande impacto na presença da $\mathrm{SM}^{7}$. Segundo Carvalho et al. ${ }^{8}$, a prevalência de SM tende a ser maior no gênero feminino e aumentar conforme a idade, assim, o que justifica a escolha do acompanhamento ser realizado com mulheres.

Nesse sentido, verifica-se a importância da alimentação como estratégia de prevenção e também tratamento da SM. Contudo não existe consenso sobre a estratégia nutricional mais adequada para tratar a $\mathrm{SM}^{9}$. Alguns autores relatam que terapias de perda de peso corporal, baseadas na redução de gordura corporal, devem envolver modificação na dieta e no nível de atividade física diária ${ }^{10,11}$.

Nesse sentido, o estudo teve como objetivo analisar a efetividade de uma estratégia nutricional em mulheres obesas com diagnóstico clínico de Síndrome Metabólica.

\section{METODOLOGIA}

Trata-se de estudo caso controle longitudinal realizado no período de junho a setembro de 2017. O estudo foi estruturado em dois grupos, sendo Grupo Caso: mulheres obesas com diagnóstico de SM e Grupo Controle: mulheres obesas sem diagnóstico de SM.

Participaram da pesquisa mulheres com diagnóstico clínico de obesidade (IMC $>30 \mathrm{~kg} / \mathrm{m}^{2}$ ), com faixa etária de 30 a 70 anos de idade, que iniciavam tratamento nutricional na Clínica Escola de Nutrição da Universidade Estadual do Centro-Oeste (Unicentro) recrutadas por meio de contato telefônico e que aceitaram participar da pesquisa mediante a assinatura do Termo de Consentimento Livre e Esclarecido (TCLE). Foram excluídas da pesquisa mulheres que estavam no período gestacional e que não realizaram acompanhamento mensal.

As avaliações ocorreram em duas etapas, sendo realizados os mesmos questionamentos para ambos os grupos. Na primeira etapa foi realizada anamnese nutricional referente a informações do paciente com foco na alimentação, antecedentes pessoais e familiares. Em seguida, foi realizada a avaliação antropométrica, com objetivo de traçar um perfil nutricional e clínico da paciente. Foram realizadas as medidas de peso $(\mathrm{kg})$, estatura $(\mathrm{m})$, circunferência do braço $(\mathrm{cm})$, circunferência da cintura CC $(\mathrm{cm})$, seguindo os protocolos do Sistema de Vigilância Alimentar e Nutricional (SISVAN) ${ }^{12}$ e sendo realizadas as classificações para definir o perfil nutricional das pacientes, incluindo: classificação do Índice de Massa Corporal $\left(\mathrm{kg} / \mathrm{m}^{2}\right)$, para adultos, segundo a Organização Mundial de Saúde 13 e para idosos, segundo a Organização Panamericana de Saúde ${ }^{14}$, classificação da $\mathrm{CB}^{15}$ e classificação da $\mathrm{CC}^{16}$.

Em primeiro contato na consulta nutricional foram repassadas orientações para melhora da qualidade da alimentação e entregue o Registro Alimentar Estimado, onde a paciente registrou todos os alimentos e bebidas consumidos durante quatro dias, sendo dias alternados e abrangendo um dia do final de semana, seguido de horários, formas de preparo e tamanho da porção consumida em medida caseira.

O segundo contato da consulta nutricional ocorreu após sete dias, em que a paciente apresentava 
o registro alimentar e logo recebia o plano alimentar. $\mathrm{Na}$ segunda etapa as participantes foram avaliadas após 30 dias ao recebimento do plano alimentar. Nessa avaliação foi realizada novamente a avaliação antropométrica. Posteriormente, foi solicitada nova realização dos mesmos exames bioquímicos solicitados anteriormente.

Todas as participantes compareceram à coleta de sangue para realização dos exames bioquímicos na Clínica Escola de Farmácia da Universidade Estadual do Centro-Oeste, que ocorreu no período da manhã. As mesmas foram submetidas à punção venosa, após jejum de 10-12 horas, para verificação dos exames bioquímicos, dentre eles: glicemia em jejum, triglicerídeos e colesterol total, os quais foram realizados em duas etapas, sendo uma etapa no início do acompanhamento e outra no término, com intervalo de 30 dias.

Para avaliação dos exames, foram utilizados os seguintes valores de referência: glicemia em jejum considerada normal apresentando valores de 65,0 a 99,0 mg/dL; elevado (pré-diabetes) de 100 a 125 mg/dL; muito elevado (diabetes mellitus) acima de $126,0 \mathrm{mg} / \mathrm{dL}^{17}$. Triglicerídeos considerado desejável com valores de 100,0 a 150,0 mg/dL; tolerável de 150,0 a 200,0 mg/dL; Elevado acima de $200,0 \mathrm{mg} / \mathrm{dL}^{18}$. Colesterol total classificado como desejável com valores abaixo de 200,0 mg/dL; Tolerável de 200,0 a 239,0 mg/dL; Elevado acima de 240,0 mg/dL ${ }^{18}$.

Foram utilizados parâmetros determinados pelo National Cholesterol Education Program's Adult Treatment Panel III (NCEP-ATP III) ${ }^{19}$ e adotado pela I Diretriz Brasileira de Diagnóstico e Tratamento da Síndrome Metabólica ${ }^{20}$, sendo determinado o diagnóstico apenas quando a paciente apresentasse a combinação de, no mínimo, três dos seguintes critérios: obesidade abdominal por meio da circunferência da cintura (CC) $\geq 88 \mathrm{~cm}$ em mulheres; triglicerídeos $\geq 150 \mathrm{mg} / \mathrm{dL}$; HDL Colesterol $<50 \mathrm{mg} / \mathrm{dL}$ em mulheres; pressão arterial $\geq 130 \mathrm{mmHg}$ ou $\geq 85 \mathrm{mmHg}$ e glicemia em jejum $\geq 100 \mathrm{mg} / \mathrm{dL}$. Ocorreram casos em que a glicemia em jejum e a pressão arterial encontravam-se controladas, pelo uso de medicamentos, mesmo assim, foram incluídos na pesquisa._

Todos os dados obtidos por meio dos inquéritos alimentares foram calculados pelo software Avanutri ${ }^{\circledR}$. Analisaram-se o consumo energético, a quantidade em gramas e o percentual de macronutrientes ${ }^{21}$, bem como análise da quantidade ingerida de gorduras saturadas, poli-insaturadas e monoinsaturadas, para posteriormente serem comparados quanto à sua adequação com os padrões de referência da Sociedade Brasileira de Cardiologia ${ }^{7}$. Além disso, incluíram-se dados referentes ao plano proposto, em que se analisaram os percentuais ofertados de carboidratos, proteínas e lipídios, bem como quantidade em gramas de colesterol e fibras.

A estratégia nutricional utilizada foi a elaboração e prescrição de um plano alimentar individualizado, para sua elaboração foram realizados os cálculos das necessidades energéticas de cada indivíduo segundo as $\mathrm{DR}^{22}$, levando em consideração idade, estatura (m), peso (kg) e nível de atividade física. Para classificação de nível de atividade física ${ }^{21}$, consideraram-se os seguintes critérios: sedentário quando realizava serviços domésticos, de esforço moderado, caminhadas para atividades do cotidiano e ficava sentado por várias horas; Atividade leve quando realizava caminhas $(6,4 \mathrm{~km} / \mathrm{h})$, além das atividades citadas anteriormente; Atividade moderada quando praticava ginástica aeróbica, corrida, natação, jogava tênis, além das atividades relacionadas ao primeiro item; e atividade intensa quando praticava ciclismo de intensidade moderada, corrida, pulava corda, jogava tênis, além das atividades realizadas pelo sedentário. Procurou-se ofertar um plano normocalórico, com adequação de macronutrientes seguindo a recomendação estabelecida pela Organização Mundial da Saúde ${ }^{21}$, em que o percentual de proteína deve equivaler de 10 a $15 \%$ do valor energético total (VET), carboidrato de 55 a 75\% e lipídeo de 15 a 30\%. Já para ácidos graxos, optou-se pela recomendação da Sociedade Brasileira de Cardiologia ${ }^{7}$, qual traz que ácidos graxos saturados devem corresponder a valores $<10 \%$ do VET, ácidos graxos monoinsaturados valores $\leq 20 \%$ do VET e ácidos graxos poli-insaturados valores $\leq 10 \%$ do VET. Para fibras, utilizaram-se valores de 20 a 30 g/dia e para colesterol $<300 \mathrm{mg} / \mathrm{dia},<200 \mathrm{mg} /$ dia (se LDL estivesse elevado) ${ }^{7}$.

Os dados foram tabulados inicialmente no $\mathrm{Ex}$ cel ${ }^{\circledR} \mathrm{e}$ analisados por meio de estatística descritiva, com médias, desvio-padrão, frequências relativas e absolutas. A distribuição das variáveis foi verificada por meio do teste de Shapiro-Wilk e Kolmogorov-Sminorv. Para comparação das variáveis quantitativas entre os grupos caso 
e controle foi utilizado o teste-T para amostras independentes (quando a distribuição era normal), ou o teste de Mann-Whitney (quando a distribuição não era normal). Para comparação das variáveis quantitativas relativas ao período inicial e final do estudo, para ambos os grupos, foi utilizado o teste-T para amostras pareadas (quando a distribuição era normal), ou teste de Wilcoxon (quando a distribuição não era normal). Para comparação das variáveis categóricas foi utilizado o teste do qui-quadrado. As análises estatísticas foram realizadas com auxílio do software SPSS versão 20.0, com nível de significância de 5\%.

O estudo foi aprovado pelo Comitê de Ética em Pesquisa (COMEP) da Universidade Estadual do Centro-Oeste, sob o parecer 2.050.954 (CAAE: 67757717.1.0000.0106).

\section{RESULTADOS}

A amostra foi constituída por 20 pacientes do sexo feminino, sendo dez no grupo Caso e dez no grupo Controle. A média de idade do grupo Caso foi de 51,50土9,23

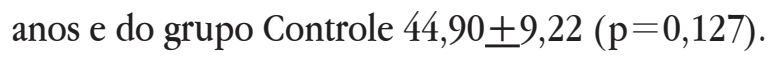

A Tabela 1 demonstra dados relacionados aos diagnósticos clínicos mais frequentes, classes de medicamentos utilizados, histórico de ganho de peso e tratamento para redução de peso, do grupo caso e controle. Observa-se que o grupo Caso apresentou maior prevalência de DCNT em relação ao grupo controle, com diagnósticos de hipertensão e dislipidemias em $90 \%(n=9)$ dos casos. Quanto às classes de medicamentos, destacou-se o uso de hipoglicemiantes orais $(70 \%, n=7)$ e anti-hipertensivos $(30 \%, \mathrm{n}=30)$ no grupo Caso. Sobre histórico de ganho de peso, notou-se relatado no período da adolescência $(40 \%, n=4)$ e após a gestação $(40 \%, n=4)$ em pacientes com SM. Observa-se que a maioria dos pacientes, em ambos os grupos, relatou já terem sido submetidos a tratamentos para perda de peso.

Tabela 1. Dados descritivos relacionados aos diagnósticos clínicos encontrados, classe de fármacos, histórico de ganho de peso e tratamento para redução dos grupos Caso e Controle

\begin{tabular}{|c|c|c|c|c|c|c|}
\hline \multirow{2}{*}{\multicolumn{2}{|c|}{ Variáveis $\mathbf{n}$}} & \multicolumn{2}{|c|}{ Grupo Caso } & \multicolumn{2}{|c|}{ Grupo Controle } & \multirow{2}{*}{$\mathbf{p}$} \\
\hline & & $\%$ & $\mathbf{n}$ & $\%$ & & \\
\hline \multirow{3}{*}{ Diagnóstico clínico } & Diabetes mellitus & 7 & 70 & 1 & 10 & 0,107 \\
\hline & Hipertensão Arterial Sistêmica & 9 & 90 & 3 & 30 & 0,490 \\
\hline & Dislipidemias & 9 & 90 & 0 & 0 & $*$ \\
\hline \multirow{5}{*}{ Fármacos } & Hipoglicemiantes orais & 7 & 70 & 1 & 10 & 0,107 \\
\hline & Anti-hipertensivos & 6 & 60 & 3 & 30 & 0,778 \\
\hline & Diuréticos & 3 & 30 & 0 & 0 & $*$ \\
\hline & Antiarrítmicos & 3 & 30 & 0 & 0 & $*$ \\
\hline & Antilipêmicos & 1 & 10 & 2 & 20 & 0,598 \\
\hline \multirow{4}{*}{ Histórico de ganho de peso } & Infância & 1 & 10 & 1 & 10 & \multirow{4}{*}{0,740} \\
\hline & Adolescência & 4 & 40 & 1 & 10 & \\
\hline & Adulta & 1 & 10 & 3 & 30 & \\
\hline & Gestação & 4 & 40 & 5 & 50 & \\
\hline \multirow{2}{*}{ Tratamento redução de peso } & Sim & 7 & 70 & 5 & 50 & \multirow{2}{*}{0,490} \\
\hline & Não & 3 & 30 & 5 & 50 & \\
\hline
\end{tabular}

Notas: $n=$ número amostral; $p=$ valor estatístico do teste; $\%=$ percentual $; *$ não se aplica teste estatístico. 
Com relação ao diagnóstico de SM, 50\% $(n=5)$ dos pacientes apresentavam no mínimo três critérios para determinação da doença, $40 \%(\mathrm{n}=4)$ apresentavam a combinação de quatro deles e apenas 10\% (n=1) apresentaram cinco critérios.

Os indicadores nutricionais avaliados na primeira etapa não tiveram diferença significante entre grupo Caso x grupo Controle. Porém, verificou-se que em ambos os grupos, maior prevalência de obesidade grau I segundo IMC, obesidade conforme aferição da $\mathrm{CB}$ e risco muito elevado para complicações metabólicas. Quanto aos exames bioquímicos, observou-se destaque no grupo Caso, em que grande parte dos pacientes encontravam-se com níveis de glicemia em jejum, colesterol total e triglicerídeos elevados. A maioria dos pacientes também apresentou consumo de calorias abaixo do recomendado, entretanto, o consumo de ácidos graxos saturados encontrava-se acima do recomendado no grupo Caso (Tabela 2).

Tabela 2. Comparação dos indicadores nutricionais, bioquímicos e alimentares de pacientes dos grupos Caso e Controle em período inicial a avaliação nutricional

(Continua)

\begin{tabular}{|c|c|c|c|c|c|c|}
\hline \multirow{2}{*}{$\begin{array}{l}\text { Indicadores } \\
\text { nutricionais }\end{array}$} & \multirow{2}{*}{ Classificação } & \multicolumn{2}{|c|}{$\begin{array}{l}\text { Grupo- } \\
\text { Caso }\end{array}$} & \multicolumn{2}{|c|}{$\begin{array}{c}\text { Grupo } \\
\text { Controle }\end{array}$} & \multirow[t]{2}{*}{ p } \\
\hline & & $\mathbf{n}$ & $\%$ & $\mathbf{n}$ & $\%$ & \\
\hline \multirow{3}{*}{$\begin{array}{c}\text { Índice } \\
\text { de Massa } \\
\text { Corporal } \\
\text { (IMC) }\end{array}$} & $\begin{array}{c}\text { Obesidade } \\
\text { grau I }\end{array}$ & 6 & 60 & 7 & 70 & \\
\hline & $\begin{array}{c}\text { Obesidade } \\
\text { grau II }\end{array}$ & 3 & 30 & 3 & 30 & 0,240 \\
\hline & $\begin{array}{l}\text { Obesidade } \\
\text { grau III }\end{array}$ & 1 & 10 & 0 & 0 & \\
\hline $\begin{array}{c}\text { Circunferência } \\
\text { da Cintura } \\
\text { (CC) }\end{array}$ & $\begin{array}{l}\text { Risco muito } \\
\text { elevado de } \\
\text { complicações } \\
\text { metabólicas }\end{array}$ & 10 & 100 & 10 & 100 & $*$ \\
\hline \multirow{3}{*}{$\begin{array}{l}\text { Circunferência } \\
\text { do Braço (CB) }\end{array}$} & Eutrofia & 3 & 30 & 1 & 10 & \multirow{3}{*}{0,22} \\
\hline & Sobrepeso & 1 & 10 & 5 & 50 & \\
\hline & Obesidade & 6 & 60 & 4 & 40 & \\
\hline \multirow{3}{*}{$\begin{array}{l}\text { Glicose em } \\
\text { jejum }\end{array}$} & Normal & 3 & 30 & 9 & 90 & \multirow{3}{*}{0,43} \\
\hline & Elevado & 3 & 30 & 1 & 10 & \\
\hline & Muito elevado & 4 & 40 & 0 & 0 & \\
\hline \multirow{3}{*}{$\begin{array}{l}\text { Colesterol } \\
\text { total }\end{array}$} & Desejável & 4 & 40 & 6 & 60 & \multirow{3}{*}{0,287} \\
\hline & Tolerável & 2 & 20 & 2 & 20 & \\
\hline & Elevado & 4 & 40 & 2 & 20 & \\
\hline
\end{tabular}

\begin{tabular}{|c|c|c|c|c|c|c|}
\hline \multirow{3}{*}{$\begin{array}{l}\text { Indicadores } \\
\text { nutricionais }\end{array}$} & \multirow{3}{*}{ Classificação } & & & \multicolumn{3}{|c|}{ (Conclusão) } \\
\hline & & \multicolumn{2}{|c|}{$\begin{array}{c}\text { Grupo- } \\
\text { Caso }\end{array}$} & \multicolumn{2}{|c|}{$\begin{array}{c}\text { Grupo } \\
\text { Controle }\end{array}$} & \multirow[t]{2}{*}{ p } \\
\hline & & $\mathbf{n}$ & $\%$ & $\mathbf{n}$ & $\%$ & \\
\hline \multirow{3}{*}{ Triglicerídeos } & Desejável & 1 & 10 & 4 & 40 & \multirow{3}{*}{0,273} \\
\hline & Tolerável & 3 & 30 & 3 & 30 & \\
\hline & Elevado & 6 & 60 & 3 & 30 & \\
\hline \multirow{2}{*}{$\begin{array}{l}\text { Adequação } \\
\text { registro ali- } \\
\text { mentar x Pla- } \\
\text { no Alimentar } \\
\text { Proposto }\end{array}$} & $\begin{array}{c}\text { Abaixo reco- } \\
\text { mendado }\end{array}$ & 7 & 70 & 7 & 70 & \multirow[b]{2}{*}{0,880} \\
\hline & $\begin{array}{l}\text { Acima reco- } \\
\text { mendado }\end{array}$ & 3 & 30 & 3 & 30 & \\
\hline $\begin{array}{l}\text { Adequação de } \\
\text { ácidos graxos } \\
\text { saturados }\end{array}$ & $\begin{array}{l}\text { Recomendado } \\
\qquad(<7 \%) \\
\text { Acima reco- } \\
\text { mendado }\end{array}$ & 7 & 30 & 6 & 60 & 0,778 \\
\hline \multirow{2}{*}{$\begin{array}{l}\text { Adequação de } \\
\text { ácidos graxos } \\
\text { monoinsatu- } \\
\text { rados }\end{array}$} & $\begin{array}{c}\text { Recomendado } \\
(\leq 20 \%)\end{array}$ & 10 & 100 & 10 & 100 & \multirow{2}{*}{$*$} \\
\hline & $\begin{array}{c}\text { Acima do } \\
\text { recomendado }\end{array}$ & 0 & 0 & 0 & 0 & \\
\hline \multirow{2}{*}{$\begin{array}{l}\text { Adequação de } \\
\text { ácidos graxos } \\
\text { poli-insatura- } \\
\text { dos }\end{array}$} & $\begin{array}{l}\text { Recomendado } \\
(\leq 10 \%)\end{array}$ & 7 & 70 & 7 & 70 & \multirow{2}{*}{0,175} \\
\hline & $\begin{array}{c}\text { Acima do } \\
\text { recomendado }\end{array}$ & 3 & 30 & 3 & 30 & \\
\hline
\end{tabular}

Notas: $n=$ número amostral; $p=$ valor estatístico do teste; $\%=$ percentual $;<=$ menor $; \leq=$ menor/igual; * não se aplica teste estatístico.

Ao analisar-se quantitativamente os indicadores nutricionais dos grupos avaliados na primeira etapa (Tabela 3), observou-se que a CC e glicemia de jejum foram superiores no grupo Caso comparado ao grupo Controle. A adequação do consumo de ácidos graxos saturados foi superior no grupo Caso, apresentando média de 9,31\% acima do recomendado comparado ao grupo Controle $(\mathrm{p}=0,018)$.

Tabela 3. Comparação dos indicadores antropométricos, bioquímicos e alimentares dos grupos Caso e Controle em período inicial ao acompanhamento nutricional

(Continua)

\begin{tabular}{|c|c|c|c|}
\hline \multirow{2}{*}{ Variáveis } & Grupo caso & Grupo controle & \multirow{2}{*}{ p } \\
\hline & Média $\pm D P$ & Média $\pm D P$ & \\
\hline Idade (anos) & $51,50 \pm 9,23$ & $44,90 \pm 9,22$ & 0,127 \\
\hline Peso (kg) & $86,70 \pm 14,53$ & $83,96 \pm 7,06$ & 0,598 \\
\hline $\begin{array}{c}\text { Índice de Massa } \\
\text { Corporal }(\mathrm{kg} / \\
\left.\mathrm{m}^{2}\right)\end{array}$ & $35,89 \pm 5,47$ & $33,19 \pm 2,36$ & 0,169 \\
\hline $\begin{array}{l}\text { Circunferência } \\
\text { da Cintura }(\mathrm{cm})\end{array}$ & $111,00 \pm 11,76$ & $98,40 \pm 5,18$ & 0,006 \\
\hline
\end{tabular}


(Conclusão)

\begin{tabular}{|c|c|c|c|}
\hline \multirow{2}{*}{ Variáveis } & Grupo caso & Grupo controle & \multirow[b]{2}{*}{$\mathbf{p}$} \\
\hline & Média $\pm D P$ & Média $\pm D P$ & \\
\hline $\begin{array}{l}\text { Circunferência } \\
\text { do Braço }(\mathrm{cm})\end{array}$ & $36,70 \pm 3,02$ & $35,60 \pm 1,73$ & 0,331 \\
\hline $\begin{array}{l}\text { Glicose em } \\
\text { jejum (mg/dL) }\end{array}$ & $138,37 \pm 64,82$ & $89,00 \pm 8,45$ & 0,028 \\
\hline $\begin{array}{c}\text { Colesterol total } \\
(\mathrm{mg} / \mathrm{dL})\end{array}$ & $223,90 \pm 42,35$ & $201,40 \pm 34,74$ & 0,210 \\
\hline $\begin{array}{l}\text { Triglicerídeos } \\
\text { (mg/dL) }\end{array}$ & $232,70 \pm 83,54$ & $171,10 \pm 78,50$ & 0,106 \\
\hline $\begin{array}{l}\text { Plano proposto } \\
\text { (kcal/dia) }\end{array}$ & $1775,30 \pm 145,16$ & $1818,85 \pm 94,22$ & 0,436 \\
\hline $\begin{array}{c}\text { Carboidrato } \\
\text { (kcal) }\end{array}$ & $229,92 \pm 9,97$ & $221,50 \pm 11,96$ & 0,615 \\
\hline Proteína (kcal) & $70,46 \pm 5,06$ & $80,13 \pm 7,99$ & 0,249 \\
\hline Lipídeo (kcal) & $224,15 \pm 18,50$ & $221,32 \pm 20,60$ & 0,665 \\
\hline $\begin{array}{l}\text { Colesterol (mg/ } \\
\text { dia) }\end{array}$ & $126,45 \pm 16,59$ & $138,77 \pm 36,08$ & 0,027 \\
\hline Fibras (g/dia) & $25,02 \pm 3,02$ & $25,85 \pm 2,33$ & 0,566 \\
\hline $\begin{array}{l}\text { Média Registro } \\
\text { Alimentar (kcal) }\end{array}$ & $1531,48 \pm 404,97$ & $1547,32 \pm 430,13$ & 0,933 \\
\hline $\begin{array}{c}\text { Carboidrato } \\
(\%)\end{array}$ & $50,10 \pm 7,27$ & $53,70 \pm 8,87$ & 0,334 \\
\hline Proteína (\%) & $21,62 \pm 5,48$ & $20,12 \pm 5,45$ & 0,547 \\
\hline Lipídeo (\%) & $28,28 \pm 6,90$ & $27,22 \pm 8,05$ & 0,735 \\
\hline $\begin{array}{l}\text { Adequação de } \\
\text { ácidos graxos } \\
\text { saturados (\%) }\end{array}$ & $9,31 \pm 3,69$ & $5,04 \pm 3,68$ & 0,018 \\
\hline $\begin{array}{c}\text { Adequação de } \\
\text { ácidos graxos } \\
\text { monoinsatura- } \\
\text { dos (\%) }\end{array}$ & $8,00 \pm 3,40$ & $7,11 \pm 3,58$ & 0,576 \\
\hline $\begin{array}{c}\text { Adequação de } \\
\text { ácidos graxos } \\
\text { poli-insatura- } \\
\text { dos (\%) }\end{array}$ & $8,55 \pm 7,09$ & $7,19 \pm 3,47$ & 0,593 \\
\hline
\end{tabular}

Notas: $D P=$ Desvio Padrão; $p=$ valor estatístico do teste.

Na segunda etapa do tratamento mantiveram-se superiores no grupo Caso comparado ao grupo Controle os valores médios da CC e glicemia de jejum $(\mathrm{p}<0,05)$. A média do colesterol total sérico também foi superior no grupo Caso com diferença marginalmente significante ao grupo Controle $(\mathrm{p}=0,059)$ (Tabela 4$)$.
Tabela 4. Comparação dos indicadores antropométricos e bioquímicos dos grupos Caso e Controle em período final ao acompanhamento nutricional

\begin{tabular}{cccc}
\hline $\begin{array}{c}\text { Indicadores } \\
\text { nutricionais }\end{array}$ & Grupo Caso & $\begin{array}{c}\text { Grupo Con- } \\
\text { trole }\end{array}$ & $\mathbf{p}$ \\
\cline { 2 - 3 } & Média \pm DP & Média \pm DP & \\
\hline Peso (kg) & $85,73 \pm 14,05$ & $83,06 \pm 6,53$ & 0,593 \\
\hline $\begin{array}{c}\text { Índice de Massa } \\
\text { Corporal }(\mathrm{kg} / \\
\left.\mathrm{m}^{2}\right)\end{array}$ & $35,52 \pm 5,10$ & $32,92 \pm 2,07$ & 0,153 \\
\hline $\begin{array}{c}\text { Circunferência } \\
\text { da Cintura (cm) }\end{array}$ & $109,00 \pm 12,45$ & $96,68 \pm 5,10$ & 0,010 \\
\hline $\begin{array}{c}\text { Circunferência } \\
\text { do Braço (cm) }\end{array}$ & $36,15 \pm 2,69$ & $34,69 \pm 1,74$ & 0,167 \\
\hline $\begin{array}{c}\text { Glicose em } \\
\text { jejum (mg/dL) }\end{array}$ & $136,70 \pm 63,16$ & $87,90 \pm 12,01$ & 0,027 \\
\hline $\begin{array}{c}\text { Colesterol total } \\
\text { (mg/dL) }\end{array}$ & $212,40 \pm 27,08$ & $182,90 \pm 37,63$ & 0,059 \\
\hline $\begin{array}{c}\text { Triglicerídeos } \\
\text { (mg/dL) }\end{array}$ & $218,90 \pm 71,98$ & $161,70 \pm 85,22$ & 0,122 \\
\hline
\end{tabular}

Notas: $D P=$ Desvio Padrão $; p=$ valor estatístico do teste.

A Tabela 5 mostra as variações dos indicadores nutricionais entre a primeira etapa e segunda etapa nos grupo Caso e no grupo Controle. Verificam-se diferenças significantes quanto às variáveis: peso $(p=0,049)$, circunferência da cintura $(\mathrm{p}=0,041)$ e níveis de triglicerídeos $(p=0,039)$, para o grupo Caso; e quanto ao grupo Controle (mulheres obesas sem diagnóstico de Síndrome Metabólica), apresentaram diferença significativa apenas as variáveis de circunferência da cintura $(\mathrm{p}=0,014)$ e circunferência do braço $(p=0,017)$. 
Tabela 5. Médias e desvio padrão de variáveis antropométricas e bioquímicas dos grupos Caso e Controle em relação a primeira e segunda avaliação após início de acompanhamento nutricional

\begin{tabular}{|c|c|c|c|c|c|c|}
\hline \multirow[t]{2}{*}{ Variáveis } & Avaliação GC 1 & Avaliação GC 2 & \multirow{2}{*}{ p } & $\begin{array}{c}\text { Avaliação GCo } \\
1 \\
\end{array}$ & $\begin{array}{c}\text { Avaliação } \\
\text { GCo } 2\end{array}$ & p \\
\hline & Média $\pm D P$ & Média $\pm D P$ & & Média土DP & Média+DP & \\
\hline Peso $(\mathrm{kg})$ & $86,80 \pm 14,60$ & $85,73 \pm 14,05$ & 0,049 & $83,96 \pm \mathbf{7 , 0 6}$ & $83,06 \pm 6,53$ & 0,113 \\
\hline Índice de Massa Corporal $\left(\mathrm{kg} / \mathrm{m}^{2}\right)$ & $35,90 \pm 5,49$ & $35,30 \pm 5,12$ & 0,051 & $33,20 \pm 2,39$ & $32,90 \pm 2,18$ & 0,081 \\
\hline Circunferência da Cintura (cm) & $111,0 \pm 11,76$ & $109,00 \pm 12,45$ & 0,041 & $98,40 \pm 5,18$ & $96,68 \pm 5,10$ & 0,014 \\
\hline Circunferência do Braço (cm) & $36,70 \pm 3,02$ & $36,15 \pm 2,69$ & 0,360 & $35,60 \pm 1,73$ & $34,69 \pm 1,74$ & 0,017 \\
\hline Glicose em jejum (mg/dL) & $138,37 \pm 64,82$ & $136,70 \pm 63,16$ & 0,268 & $89,00 \pm 8,45$ & $87,90 \pm 12,01$ & 0,705 \\
\hline Colesterol total (mg/dL) & $223,90 \pm 42,35$ & $212,40 \pm 27,08$ & 0,087 & $201,40 \pm 34,74$ & $182,90 \pm 37,63$ & 0,151 \\
\hline Triglicerídeos (mg/dL) & $232,70 \pm 83,54$ & $218,90 \pm 71,98$ & 0,039 & $171,10 \pm 78,50$ & $161,70 \pm 85,22$ & 0,316 \\
\hline
\end{tabular}

Notas: DP = Desvio Padrão; GC = Grupo Caso; GCo = Grupo Controle; $p=$ valor estatístico do teste.

\section{DISCUSSÃO}

Incluiu-se no estudo apenas o sexo feminino, pela prevalência de SM acometer mais as mulheres do que homens, principalmente com o decorrer da idade ${ }^{8}$. De fato, estudos demonstram a relação entre os gêneros. Kin et al. ${ }^{23}$ constataram que a maior prevalência de SM ocorre na faixa etária acima de 50 anos em mulheres, sugerindo que a fase de transição menopáusica da mulher possa ser considerada um fator determinante para aumento da mesma.

Todas as participantes dos grupo Caso e Controle encontravam-se com diagnóstico nutricional de obesidade e, consequentemente, apresentavam a CC elevada, demonstrando que a gordura se localiza na região abdominal em ambos os casos, característico de gordura androide, sendo indicativo para risco de complicações metabólicas associadas à obesidade, tais como diabetes mellitus, hipertensão arterial sistêmica, dislipidemias, doenças coronarianas, entre outras ${ }^{24}$.

Apesar de o grupo Controle não apresentar diagnóstico de SM, a presença de uma ou outra comorbidade associada já se torna um risco para desenvolvimento da mesma $^{25}$. No estudo de Ogbera et al. ${ }^{26}$, encontrou-se relação entre SM e CC, em que $80 \%$ dos pacientes diabéticos possuíam diagnóstico de SM e apresentavam alterações na circunferência da cintura, resultante de uma dieta inadequada e ausência de atividade física. Wang et al. ${ }^{27}$ verificaram correlações significativas entre parâmetros de gordura abdominal e biomarcadores metabólicos como glicemia em jejum, pressão arterial sistêmica, e dislipidemias, quais se destacaram como principais ferramentas para diagnóstico de SM.

A maioria dos pacientes do grupo Caso $(90 \%$, $\mathrm{n}=9$ ) apresentavam diagnóstico clínico de hipertensão e dislipidemias, porém nem todos faziam o uso de medicamento anti-hipertensivo e hipolipemiantes. Segundo Lira et $a l .^{28}$, realizar o controle de pressão arterial e de dislipidemias reduz as chances de complicação macro e microvasculares, diminuindo a possibilidade de acidente vascular cerebral e fatores de risco para desenvolvimento de doenças cardiovasculares.

Salienta-se que conforme relato das participantes, a maioria dessas referiu já ter iniciado algum tratamento para redução de peso. Segundo Teixeira et al.$^{29}$, as principais causas para falha na adesão são a falta de tempo, a insatisfação com a estratégia utilizada, os problemas pessoais e as limitações de saúde. Por conta disso, a estratégia nutricional utilizada levou em consideração vários fatores, tais como individualidade do paciente (sexo, idade, estado civil, escolaridade, nível socioeconômico), particularidades da doença a qual relatou (cronicidade, sintomas), tratamento medicamentoso, hábitos alimentares e culturais, buscando maior eficácia da intervenção entre os grupos.

Quando comparamos os indicadores antropométricos, bioquímicos e alimentares do grupo Caso com o grupo Controle em início de tratamento, é nítido que pacientes com diagnóstico de SM apresentem valores maiores para circunferência da cintura, glicemia em jejum e 
colesterol total, em decorrência dos critérios utilizados para sua estratificação.

A maioria dos pacientes de ambos os grupos quando iniciaram o tratamento nutricional evidenciou baixo consumo calórico comparado às necessidades nutricionais adequadas a cada faixa etária. Barbieri et al. ${ }^{30}$ constataram que apesar da ingestão ser reduzida em pacientes obesos, este método não garante a perda de peso, pois os mesmos apresentam gasto energético reduzido, proveniente do sedentarismo ${ }^{31}$.

A estratégia de reeducação alimentar busca obter influência nos hábitos alimentares, facilitando a procura por alimentos mais saudáveis, sem diminuir extremamente a oferta de calorias, sendo um método seguro e de mais fácil adesão, visando a redução de peso lenta e gradual ${ }^{32}$. Além disso, estudos demonstram que uma redução ponderal de 5 a 10\% do peso já é suficiente para proporcionar melhora significativa das medidas antropométricas e, consequentemente, nos parâmetros bioquímicos, apresentando eficácia da estratégia no tratamento de DCNT ${ }^{33,34}$.

No estudo a ingestão média do Registro Alimentar do grupo Caso para proteína foi de 21,62\% do GET, para carboidrato $50,10 \%$ e para lipídio $28,28 \%$, sendo semelhantes os valores para o grupo Controle. Evidencia-se que ambos os grupos apresentam erros quanto à distribuição de macronutrientes, consumindo quantidades acima do recomendado para proteínas. A ingestão excessiva de proteínas pode elevar as concentrações sanguíneas de colesterol, de forma independente ou conjunta ${ }^{35}$. Porém, a média de valores para colesterol total dos grupos Caso e Controle em início de tratamento nutricional encontravam-se "toleráveis" entre 223,90 mg/dL $( \pm 42,35)$ e 201,40 mg/dL (土34,74).

Por outro lado, o estudo revelou que pacientes com SM apresentavam ingestão acima do recomendado para ácidos graxos saturados antes de aderirem ao tratamento, podendo ser observado pelo registro alimentar a presença de alimentos de origem animal, como a manteiga, carnes gordurosas, banha de porco, leites e derivados, quais podem causar danos à saúde, propiciando o desenvolvimento de dislipidemias, resultando em níveis de LDL aumentados ${ }^{36}$. Ricarddi et al..$^{37}$ evidenciaram que uma alimentação rica em ácidos graxos saturados atua desfavorecendo a resistência à insulina, causando maior estresse oxidativo. Assim, pode-se notar que o grupo Caso apresentou valores de glicemia em jejum maiores comparados ao grupo Controle desde o início do tratamento nutricional.

Verificou-se melhora do perfil glicêmico, principalmente no grupo Caso. De acordo com Jansen ${ }^{38}$, o controle da glicemia demonstra relação direta com a qualidade de vida de pacientes diabéticos. Desta forma, a intervenção nutricional proposta trouxe benefícios, diminuindo valores glicêmicos, bem como perda de peso, proveniente de melhor porcionamento das refeições e adequadas escolhas de alimentos.

Pode-se observar que as variáveis de peso, circunferência da cintura e triglicerídeos alteraram-se de maneira positiva quando comparada ao início de tratamento, demonstrando eficácia da estratégia nutricional em pacientes com SM. O estudo de Bernandes ${ }^{39}$ também demonstrou alterações quanto aos parâmetros antropométricos com redução de peso e circunferência da cintura, além disso, obteve reduções significantes de glicemia, colesterol total e pressão arterial. Segundo Banegas et al. ${ }^{40}$, estima-se que o aumento de 4,5 na circunferência da CC em mulheres, eleve $1 \mathrm{mmHg}$ na PA sistólica, ocasionando maiores riscos aos pacientes com SM.

Já para grupo Controle, houve redução quanto aos parâmetros antropométricos de circunferência da cintura e do braço em relação a primeira e segunda avaliação após início do acompanhamento nutricional, demonstrando que pode haver perda de medidas antropométricas sem que haja exclusivamente perda de peso.

O presente estudo apresentou limitações quanto ao tempo de acompanhamento, sendo um período curto para possíveis mudanças, porém, pensando que estratégia de reeducação alimentar busca perda de peso mais lenta e gradual. Outra limitação foi a abordagem do consumo alimentar por meio de análise do registro alimentar, não sendo um bom indicador para representar a dieta habitual. Entretanto, foi o método encontrado de mais fácil aplicabilidade em decorrência do tempo reservado para o estudo. Além disso, houve restrição por não haver a disponibilidade para realização do exame bioquímico para colesterol HDL, considerando que o mesmo é um dos critérios para classificação da SM, contudo, obti- 
veram-se bons resultados com os exames de rotina do acompanhamento nutricional.

O estudo demonstrou resultados positivos em ambos os grupos, porém a efetividade foi maior em pacientes com SM, pois os mesmos apresentaram mudanças nos hábitos alimentares, resultando em perda de peso, diminuição de medidas antropométricas e alterações nos exames laboratoriais, em decorrência da estratégia de reeducação alimentar a qual foi proposta no início do tratamento. Portanto, conclui-se que a intervenção nutricional, por meio do planejamento alimentar foi efetiva em curto prazo. Assim verifica-se o quão relevante é o acompanhamento nutricional, podendo auxiliar na minimização e controle das comorbidades presentes na SM. Porém, é necessário ainda estudos que busquem avaliar relativamente a qualidade da alimentação e assim realizar intervenções nutricionais em longo prazo, para garantir um tratamento mais eficaz e a prevenção de DCNT.

\section{REFERÊNCIAS}

1. Martin K, Mani M, Mani A. New targets to treat obesity and the metabolic syndrome. Eur J Pharmacol. 2015; 15;763 (Pt A):64-74.

2. Kaur J. A comprehensive review on Metabolic Syndrome. Cardiol Res Pract. 2014; 2014:943162.

3. Neto JCGL, Xavier MA, Borges JWP, Araújo MFM, Damasceno MMC, Freitas RWJF. Prevalence of Metabolic Syndrome in individuals with Type Diabetes Mellitus. Rev Bras Enferm. 2017; 70(2):265-70.

4. Gami AS, Witt BJ, Howard DE, Erwin PJ, Gami LA, Somers VK, Montori VM. Metabolic syndrome and risk of incident cardiovascular events and death: a systematic review and meta-analysis of longitudinal studies. J Am Coll Cardiol. 2007;49(4):403-14.

5. Junges VM, Cavalheiro JMB, Fam EF, Closs VE, Gottlieb MGV. Perfil do candidato obeso e portador da síndrome metabólica candidato à cirurgia bariátrica em uma clínica particular de Porto Alegre, Rio Grande do Sul. Sci Med. 2016;26(3).

6. The World Health Report 2002: Reducing Risks, Promoting Healthy Life. Genebra: WHO, 2002.
7. Sociedade Brasileira de Cardiologia. I Diretriz Brasileira de diagnóstico e tratamento da Síndrome Metabólica. SBC. 2005; 84:1-28.

8. Carvalho Vidigal, F, Bressan, J, Babio, N, Salas-Salvadó, J. Prevalence of metabolic syndrome in Brazilian adults: a systematic review. BMC Public Health. 2013; 13(1), 1198.

9. Leão LSCD, Moraes MM, Carvalho GX, Koifman RJ. Nutritional interventions in Metabolic Syndrome. A systematic review. Arq Bras Card. 2011; 97(3):260265.

10. Carneiro, D. Prescrição do exercício físico: a sua inclusão na consulta. Rev. Port. Clin. Geral. 2011;27(5):470-9.

11. Lopes JF, Matos MA, Magalhães FC, Esteves EA, Vieira ET, Amorim FT. Efeito de mudanças graduais de exercícios físicos e dieta sobre a composição corporal de obesos. Arq. Ciênc. Saúde. 2017; 24(1):93-97.

12. Brasil. Ministério da Saúde. Secretaria de Atenção à Saúde. Departamento de Atenção Básica. Orientações para a coleta e análise de dados antropométricos em serviços de saúde: Norma Técnica do Sistema de Vigilância Alimentar e Nutricional - SISVAN / Ministério da Saúde, Secretaria de Atenção à Saúde, Departamento de Atenção Básica. - Brasília: Ministério da Saúde, 2011.

13. World Health Organization. Physical status the use and interpretation of anthropometrics. Report of a World Health Organization. Expert Committee. WHO. Tech Rep Ser. 1995; 854(1):1-452.

14. Organização Pan-Americana de Saúde - OPAS/OMS, SABE - Saúde, Bem Estar e Envelhecimento. O projeto SABE no Município de São Paulo: uma abordagem inicial. $1^{\mathrm{a}}$ ed. São Paulo: Athalaia Bureau, 2003.

15. Frisancho AR. Anthropometric Standars for the Assessment of Growth and Nutritional Status. University of Michigan; 1990.

16. World Health Organization. Obesity: preventing and managing the global epidemic. Geneva: World Health Organization; 1998. (WHO Technical Report Series 894).

17. American Diabetes Association. Gestational diabetes mellitus. Diabetes Care. 2004; 27 Suppl 1:S88-90.

18. Sociedade Brasileira de Análises Clínicas. Consen- 
so Brasileiro para a Normatização da Determinação Laboratorial do Perfil Lipídico. Rio de Janeiro, dez. 2016.

19. National Institutes of Health. Clinical guidelines on the identification, evaluation, and treatment of overweigth and obesity in adults. In: National Institutes of Health National Heart Lung and Blood Institute, editors, 1998.

20. Sociedade Brasileira de Hipertensão. Sociedade Brasileira de Cardiologia. Sociedade Brasileira de Endocrinologia e Metabologia. Sociedade Brasileira de Diabetes. Associação Brasileira para Estudos da Obesidade. I Diretriz brasileira de diagnóstico e tratamento de Síndrome Metabólica. Arq Bras Cardiol. 2005; 84(supl 1):1-28.

21. World Health Organization. Food and Agriculture Organization [WHO/FAO]. Diet, nutrition and the prevention of chronic diseases. Geneva; 2003. [WHO - Technical Report Series, 916]

22. Institute of Medicine. Dietary reference intakes for energy, carbohydrate, fiber, fat, fatty acids, cholesterol, protein, and amino acids. Washington (DC): National Academy Press; 2002.

23. Kim HM, Park J, Ryu S. Y, Kim J. The effect of menopause on the metabolic syndrome among Korean women: the Korean National Health and Nutrition Examination Survey, 2001. Diabetes Care, 30(3):701-06.

24. Bortoli C, et al. Ingestão Dietética de Gordura Saturada e Carboidratos em Adultos e Idosos com Dislipidemias Oriundos do Projeto Veranópolis. Rev Bras Cardiol. 2011;24(1):33-4.

25. Assunção WAC, Prado WLP, Oliveira LMFT, Falcão APST, Costa MC, Guimarães FJSP. Comportamento da gordura abdominal em mulheres com avanço da idade. Rev. Educ. Fis/UEM. 2013;24(2):287-294

26. Ogbera AO. Prevalence and gender distribution of the metabolic syndrome. Diabetol Metab Syndr. 2010;2:1.

27. Wang X, Strizich G, Hua S, Sotres-Alvarez D, Buelna C, Gallo LC, Gellman MD, Mossavar-Rahmani Y, O'Brien MJ, Stoutenberg M, et al. Objectively Measured Sedentary Time and Cardiovascular Risk Factor Control in US Hispanics/Latinos With Diabetes Mellitus: Results From the Hispanic Community
Health Study/Study of Latinos (HCHS/SOL). J. Am. Heart Assoc. 2017.

28. Lira AM, Athaydes BR, Provedel G, Gonçalves NAZ, Sales CMM, Guimarães do Bem DAM et al. Síndrome Metabólica em Diabéticos tipo 2 em Insulinoterapia: Fatores associados e Qualidade de Vida Journal of Applied Pharmaceutical Sciences - JAPHAC, 2016; 3(3): 15-22.

29. Teixeira PDS, Reis BZ, Vieira DAS, Costa D, Costa GO, Raposo OFF, et al. Intervenção nutricional educativa como ferramenta eficaz para mudança de hábitos alimentares e peso corporal entre praticantes de atividade física. Ciência da Saúde Coletiva. 2013; 18(2): 347-356.

30. Barbieri AF, Mello RA. As causas da obesidade: uma análise sob a perspectiva materialista histórica. Revista da Faculdade de Educação Física da UNICAMP. 2012;10(1):133-153.

31. Maki-Nunes C, Toschi-Dias E, Cepeda FX, Rondon MU, Alves MJ, Fraga RF, Braga AM, Aguilar AM, Amaro AC, Drager LF. Diet and exercise improve chemoreflex sensitivity in patients with metabolic syndrome and obstructive sleep apnea. Obesity (Silver Spring). 2015, 23(8) 1582-90.

32. Moreira P, Romualdo MCS, Amparo FC, Paiva C, Alves R, Magnoni D, Kovacs C. A educação nutricional em grupo e sua efetividade no tratamento de pacientes obesos. Revista Brasileira de Obesidade, Nutrição e Emagrecimento. 2012, 6(35): 216-224.

33. Sociedade Brasileira de Cardiologia. V Diretrizes Brasileiras de Hipertensão Arterial. Arq Bras Cardiol 2007; 89(3): 24-78.

34. Piantino CB, Cunha JC, Amâncio MCS, Almeida JC, Pádua KM. Parâmetros bioquímicos: Associação com o estado nutricional e presença de patologia. Revista Brasileira de Obesidade, Nutrição e Emagrecimento, São Paulo. 2017; 11(62): 86-93.

35. Ramos CO, Gomes AS, Previato HDRA, Volp ACP. Associação entre medidas antropométricas, de composição corporal, bioquímicas e de consumo alimentar em indivíduos com excesso de peso. Rev Bras Promoc Saude. 2013;26(2):157-65.

36. Lottenberg, AM, Afonso MS, Lavrador MSF, Machado RM, Nakandakare ER. The role of dietary fatty acids in the pathology of metabolic syndrome. Journal of 
Nutritional Biochemistry 23 (2012) 1027-1040.

37. Riccardi G, Giacco R, Rivellese AA. Dietary fat, insulin sensitivity and the metabolic syndrome. Clin Nutr. 2004; 23(4):447-56.

38. Jansen HJ, Stienstra R, van Diepen JA, Hijmans A, van der Laak JA, Vervoort GM, Tack CJ. Start of insulin therapy in patients with type 2 diabetes mellitus promotes the influx of macrophages into subcutaneous adipose tissue. Diabetologia. 2013;56(12):257381.

39. Bernardes MS. Atendimento nutricional coletivo como estratégia de ação para o tratamento do excesso de peso em mulheres adultas na estratégia da família em Poços de Caldas - MG. Campinas SP: 2016.

40. Benegas JR, Lopez GE, Dallongeville J, Guallar E, Borghi C, Masso GEL, Jimenez FJ, Perk J, Steg PG, Backer $G$, Rodrigues AF. Achievement of treatment goals for primary prevention of cardiovascular disease in clinical practice across. Europe: the EURIKA study. Eur Heart J, 2011, 32(17): 2143-2152.

Recebido em: 03/10/2018

Aceito em: 21/05/2019 